\title{
PROPOSTA DE INSTRUMENTO PARA DIAGNÓSTICO DA GESTÃO DO CONHECIMENTO NO ARSENAL DE MARINHA DO RIO DE JANEIRO
}

\author{
Lívia de Souza Ribeiro \\ Universidade Federal Fluminense \\ Rua Passo da Pátria, 156 - Bloco E - São Domingos - Niterói - CEP:24210-240 \\ liribeiro.86@gmail.com \\ Stella Regina Reis da Costa \\ Universidade Federal Fluminense \\ Rua Passo da Pátria, 156 - Bloco E - São Domingos - Niterói - CEP:24210-240 \\ stellare@ig.com.br
}

\section{RESUMO}

As organizações contemporâneas estão inseridas em um contexto de rápidas e constantes mudanças nos quesitos político, econômico e tecnológico. Neste cenário, a gestão do conhecimento (GC) para a efetiva tomada de decisões e inovação é fundamental para a sustentabilidade das organizações assim como para a manutenção de vantagens competitivas. Nas organizações públicas, a gestão do conhecimento é base para reduzir os efeitos da perda de capital humano, em especial nos casos de aposentadoria e movimentação. Diante disto, este estudo tem como objetivo geral propor um instrumento para diagnóstico da gestão do conhecimento no Arsenal de Marinha do Rio de Janeiro, que é uma organização militar da Marinha do Brasil. Para alcançar o referido objetivo, será utilizada como metodologia uma pesquisa bibliográfica, qualitativa e de natureza exploratória. Como resultado deste estudo está a proposta de um instrumento para diagnóstico da GC na organização no formato de um questionário com embasamento teórico. A partir deste instrumento, poderá ser realizada a identificação da importância da GC na organização, identificação das principais dificuldades para a implantação da GC na organização e a identificação das práticas mais adaptáveis à organização assim como as já existentes.

Palavra-chave: Gestão do conhecimento; Práticas de gestão do conhecimento; Ferramentas de gestão do conhecimento; Administração pública; Setor público.

\begin{abstract}
Contemporary organizations are inserted in a context of rapid and constant changes in the political, economic and technological aspects. In this scenario, knowledge management (KM) for effective decision making and innovation is fundamental for the sustainability of organizations as well as for maintaining competitive advantages. In public organizations, knowledge management is the basis for reducing the effects of loss of human capital, especially in the case of retirement and movement. Given this, this study aims to propose a tool for the diagnosis of knowledge management in the Navy Arsenal of Rio de Janeiro, which is a military organization of the Brazilian Navy. To achieve this objective, a bibliographical, qualitative and exploratory research will be used as methodology. As a result of this study is the proposal of a tool for diagnosis of KM in the organization in the form of a theoretical basis questionnaire. From this instrument, it will be possible to identify
\end{abstract}


the importance of $\mathrm{KM}$ in the organization, identify the main difficulties for the implementation of KM in the organization and identify the most adaptable practices to the organization as well as the existing ones.

Keywords: Knowledge management; Knowledge management practices; Knowledge management tools; Public administration; Public sector.

\section{Como Citar:}

RIBEIRO, Lívia de Souza; COSTA, Stella Regina Reis da. Proposta de instrumento para diagnóstico da gestão do conhecimento no Arsenal de Marinha do Rio de Janeiro. In: SIMPÓSIO DE PESQUISA OPERACIONAL E LOGÍSTICA DA MARINHA, 19., 2019, Rio de Janeiro, RJ. Anais [...]. Rio de Janeiro: Centro de Análises de Sistemas Navais, 2019.

\section{INTRODUÇÃO}

\subsection{CONTEXTUALIZAÇÃO}

Com o nível de competição entre organizações cada vez maior, a gestão das organizações contemporâneas precisa se adaptar a mudanças cada vez mais rápidas no mercado global. Desta forma, a busca por vantagens competitivas que aumentem o nível de excelência de produtos ou serviços e gerem valor diferencial para os clientes é fator vital para estas organizações.

Mudanças nos cenários político, econômico, social e tecnológico podem interferir diretamente na manutenção de vantagens competitivas se estas forem pontuais. Uma mudança tecnológica pode rapidamente transformar uma vantagem pontual em obsoleta. Neste sentido, o conhecimento aplicado no contexto organizacional para a solução de problemas não se desgasta, mas se renova potencializando o desenvolvimento organizacional. (CARBONE et al., 2009)

Segundo Rocha et al. (2012), O conhecimento é considerado um dos ativos mais importantes nas organizações, pois é capaz de apoiar as decisões organizacionais e individuais de forma mais inteligente, eficiente e eficaz, estimulando continuamente a inovação em produtos e serviços. Entretanto, De Angelis (2016) considera necessária a mudança da cultura "conhecimento é poder" para "compartilhar conhecimento é poder" pois o conhecimento que não é compartilhado é perdido.

Nas organizações públicas, a aplicação efetiva da gestão do conhecimento é capaz de reduzir os efeitos da perda de capital humano. (PEE; KANKANHALLI, 2016)

Para Nonaka e Takeuchi (2008), gerenciar o conhecimento de forma a criar ou manter vantagem competitiva tornou-se foco central das estratégias de competição de muitas indústrias.

\subsection{SITUAÇÃO PROBLEMA}

O Arsenal de Marinha do Rio de Janeiro (AMRJ) é a mais antiga Organização Militar (OM) da Marinha do Brasil (MB) sendo o estaleiro mais importante de reparo e manutenção de meios navais da MB.

Apesar de ser um estaleiro militar, a mão de obra utilizada no AMRJ sempre foi em sua maior parte civil. O conhecimento tácito era compartilhado naturalmente nas atividades do dia a dia. A permanência da mão de obra técnica de referência, e a quantidade grande de projetos em andamento permitia aos funcionários mais novos o acompanhamento e aprendizado in loco, apoiados pelos mais experientes e conhecedores técnicos. 
A mão de obra civil detentora de conhecimentos técnicos relevantes está se tornando escassa. Em relação aos funcionários públicos civis, em virtude da não realização de novos concursos capazes de repor a mão de obra perdida face aos processos de aposentadoria. Observa-se ainda a redução do efetivo de funcionários civis contratados.

Diante disso, a necessidade de substituição desta mão de obra por militares assim como a terceirização de serviços não considerados críticos e/ ou estratégicos é a diretriz para que o AMRJ continue exercendo sua missão. Entretanto, algumas dificuldades relevantes surgem no campo da gestão do conhecimento para a manutenção da qualidade dos serviços realizados.

Um dos principais desafios da OM será estabelecer uma gestão do conhecimento de forma estruturada e institucionalizada em uma Organização Militar, que em virtude da própria carreira dos militares tem a mobilidade geográfica como característica da força de trabalho, de forma a permitir o desenvolvimento, compartilhamento e retenção do conhecimento seja realizado para que o AMRJ continue exercendo seu compromisso institucional com qualidade.

\subsection{OBJETIVO GERAL}

O objetivo deste artigo é propor um instrumento de diagnóstico para a gestão do conhecimento na OM com base na revisão de literatura.

\section{METODOLOGIA}

A metodologia utilizada na classificação de Gil (2010) será de natureza exploratória, no que se refere aos objetivos, pois constitui-se de uma investigação visando proporcionar conhecimento mais aprofundado do tema e, quanto aos meios utilizados, será no formato de pesquisa bibliográfica constituindo-se, principalmente de artigos científicos e livros. Para Fink (2005), a pesquisa bibliográfica é um método sistemático que permite identificar, avaliar e sintetizar um trabalho registrado produzido por outros pesquisadores. Segundo Gray (2012) a revisão bibliográfica demonstra as teorias, os argumentos e as polêmicas essenciais no campo e destaca as formas como a pesquisa na área foi realizada por outras pessoas.

O instrumento de pesquisa (questionário), objetivo deste estudo, será formulado com embasamento teórico a partir da pesquisa bibliográfica.

Para a coleta de dados referente a pesquisa bibliográfica foram utilizados artigos científicos, livros e dissertações. Foi utilizada a base de dados Scopus disponível no portal da Coordenação de Aperfeiçoamento de Pessoal de Nível Superior (CAPES). Segundo Singuenza-Guzman et al. (2015), Scopus é a base de dados de produção científica que oferece a melhor cobertura de artigos de periódicos identificados na área multidisciplinar. Foram utilizados 2 formatos de pesquisa conforme descrição a seguir:

\begin{tabular}{|c|c|c|}
\cline { 2 - 3 } \multicolumn{1}{c|}{} & Pesquisa 1 & Pesquisa 2 \\
\hline Data da pesquisa & $26 / 10 / 2018$ & $05 / 11 / 2018$ \\
\hline Termos utilizados & $\begin{array}{c}\text { "Knowledge management" AND } \\
\text { ("public administration" OR } \\
\text { "public sector") }\end{array}$ & $\begin{array}{c}\text { "Knowledge management practices" } \\
\text { OR "Knowledge management tools" }\end{array}$ \\
\hline Ano de publicação & 2009 a 2018 & 2009 a 2018 \\
\hline Tipo de documento & Artigo & Artigo \\
\hline RESULTADO & 228 artigos & 461 artigos \\
\hline
\end{tabular}

Quadro 1: Método de Pesquisa Bibliográfica

Fonte: As autoras 
Apenas 10 artigos se repetiram nas duas pesquisas (interseção entre a pesquisa 1 e a pesquisa 2). Com esses artigos identificados foi realizada a leitura dos títulos e resumos com o objetivo de selecionar os artigos relevantes para o contexto da atual pesquisa. Da pesquisa 1 foram selecionados 53 artigos de interesse. Da pesquisa 2 foram selecionados 79 artigos de interesse. Foram encontrados 3 artigos em comum nas duas seleções. Desta forma, totalizaram 129 diferentes artigos para leitura.

Adicionalmente, foi realizada pesquisa no Repositório Institucional da Universidade Federal Fluminense (RIUFF) no dia 11/10/2018 a partir do termo "gestão do conhecimento". Foram gerados 125 resultados para dissertações dos quais 6 dissertações foram selecionadas.

Os artigos e dissertações selecionados além de compor o referencial teórico foram direcionadores para outros materiais de autores relevantes na temática de gestão de conhecimento.

\section{REVISÃO DA LITERATURA}

\subsection{GESTÃO DO CONHECIMENTO NAS ORGANIZAÇÕES}

Para Nonaka e Takeuchi (2008), o conhecimento está relacionado a crenças do seu portador e a ação. Também está vinculado a uma finalidade. Da mesma forma, De Angelis (2016) acredita que a capacidade de transformar a informação em conhecimento está relacionado diretamente às capacidades humanas de interpretar, integrar, analisar e atuar com um propósito.

O conhecimento é dividido em duas dimensões: tácito e explícito. O conhecimento tácito, que inclui elementos cognitivos e técnicos é pessoal, difícil de codificar e transferir. Já o conhecimento explícito pode ser transferido na linguagem formal, codificado de forma sistemática. (NONAKA; TAKEUCHI, 2008; POLANYI, 1966)

Para Nonaka e Takeuchi (2008), a parte técnica do conhecimento tácito engloba as habilidades informais e de difícil detecção relacionado a experiência e ao saber fazer . A parte cognitiva do conhecimento tácito faz referência a crenças, percepções, valores e modelos mentais.

Mesmo com características distintas, as dimensões tácitas e explícitas do conhecimento precisam ser gerenciadas para que o conhecimento dos indivíduos se torne o conhecimento da organização.

Para Stewart (1998), mesmo com a dificuldade de codificação o conhecimento tácito precisa ser explicitado para que não se perca e que possa ser analisado, aperfeiçoado e mais facilmente compartilhado.

Segundo Choo (2003), o conhecimento tácito é difícil de verbalizar e codificar pois é centrado na experiência do indivíduo. Mas apesar desta dificuldade, o conhecimento tácito é compartilhado por meio do exemplo, como os aprendizes que aprendem por meio do exemplo dos mestres.

Para Nonaka e Takeuchi (2008), uma organização cria e utiliza o conhecimento através da constante interação entre os conhecimentos tácito e explícito a partir de quatro modelos de conversão de conhecimento (conhecido como processo SECI): socialização (tácito para tácito), externalização (tácito para explícito), combinação (explícito para explícito) e internalização (explícito para tácito).

A socialização utiliza a base da partilha de experiências para a transferência de conhecimento. A socialização, se incentivada e agregada a cultura da organização, permite o compartilhamento dos conhecimentos através de diversas práticas de GC. (AZEREDO; COSTA, 2018)

Na externalização o conhecimento tácito é transformado em explícito através da 
utilização de palavras e códigos. Segundo Choo (2003), No processo de externalização ocorre o compartilhamento do conhecimento tácito através de modelos, metáforas, analogias ou histórias.

Na combinação é criado conhecimento explícito novo. A combinação de conhecimentos envolve a combinação de bases diferentes de conhecimentos explícitos por meio de documentos, reuniões, conversas, entre outros. (NONAKA; TAKEUCHI, 2008)

Para Nonaka e Takeuchi (2008), na internalização ocorre a incorporação do conhecimento explícito externalizado para conhecimento tácito do indivíduo.

Gerir o conhecimento é sistematizar as práticas de conversão de conhecimento entre tácito e explícito e do indivíduo, para grupos até alcançar o nível da organização. (NONAKA; TAKEUCHI, 2008).

De Angelis (2013) define a gestão do conhecimento como um conjunto de práticas que permitem a interação de conhecimentos tácitos e explícitos objetivando o desenvolvimento de novas competências o que permite que as organizações funcionem de forma mais inteligente.

Para Chawla e Joshi (2010), a gestão do conhecimento pode ser entendida como criação, análise, armazenamento, disseminação e utilização do conhecimento como forma de apoiar a tomada de decisão e a aprendizagem contínua.

Para Handzic (2011), o objetivo prioritário da gestão do conhecimento é encontrar as formas mais eficientes de explorar e desenvolver o conhecimentos dos níveis individuais aos da organização.

\subsection{GESTÃO DO CONHECIMENTO NAS ORGANIZAÇÕES PÚBLICAS}

Segundo Amayah (2013), a natureza burocrática de várias organizações governamentais não é propícia ao compartilhamento e a aplicação de práticas efetivas de gestão do conhecimento. A estrutura rígida e hierarquizada da organização também dificulta o fluxo de conhecimento entre os diversos setores. Nonaka e Takeuchi (2008) corroboram com esta ideia ao afirmar que a hierarquia não é adequada para a gestão de conhecimentos tácitos, pois pode prejudicar a iniciativa individual a partir do controle fortemente exercido característico de ambientes hierarquizados.

Para Ribeiro et al. (2011), as instituições governamentais produzem muitas informações entretanto, em muitos casos, não as organizam ou disponibilizam de forma adequada para que estas habilitem a criação do conhecimento e apoiem de forma eficaz a tomada de decisão.

Apesar destas dificuldades, o conhecimento, assim como nas organizações privadas, é o recurso central das organizações do setor público. Diante disso, uma efetiva gestão do conhecimento nas instituições públicas é essencial para a melhoria da prestação de contas à sociedade e para um melhor nível de serviço público. (KIM; LEE, 2010)

A efetiva aplicação da gestão do conhecimento atende a um dos grandes desafios de diversas organizações do setor público, que é a perda de capital humano, nem sempre com a devida reposição e a rotatividade de pessoal. (PEE; KANKANHALLI, 2016)

Para Chong et al. (2011) a rotação no trabalho, que é uma prática comum nas organizações públicas onde os trabalhadores são transferidos ou rotacionados com frequência, é considerada de forma positiva para o desempenho organizacional. A experiência em diversos setores obtida com a movimentação é um fator potencial para a criação de conhecimentos. Por outro lado, Salleh (2013) considera que a rotação apesar de ser positiva para o desempenho organizacional, dificulta o compartilhamento dos conhecimentos tácitos, não sendo apropriado para um ambiente eficaz no que se refere a gestão do conhecimento.

Para Campos e Baptista (2011), a maior barreira para a gestão do conhecimento nas 
organizações públicas é a questão da resistência a mudanças, o que é apenas um reflexo da cultura organizacional. Para Nonaka e Takeuschi (2008), as pessoas tendem a ser reativas em adotar novos conhecimentos que contrariem suas histórias e experiências, principalmente se for transmitidos por outros grupos. Segundo Bem et al. (2013), os desafios a implementação da gestão do conhecimento em organizações públicas são grandes em virtude das barreiras culturais tais como fatores burocráticos, estabilidade e descontinuidade administrativa.

A mudança do cenário político também influencia diretamente as organizações públicas. A mudança da estratégia organizacional para a adequação das diretrizes governamentais é fator relevante que pode influenciar negativamente uma efetiva e sustentável gestão do conhecimento na organização.

Para Batista (2012), muitas organizações públicas aplicam práticas de gestão do conhecimento que não estão alinhadas com a estratégia organizacional, desta forma acabam não contribuindo para o alcance de objetivos estratégicos da organização o que , por muitas vezes, atribui a gestão do conhecimento um papel secundário no desempenho organizacional.

\subsection{FACILITADORES E BARREIRAS PARA A APLICAÇÃO DA GESTÃO DO CONHECIMENTO.}

A partir da pesquisa bibliográfica, foram elencados fatores citados por diversos autores como habilitadores ou facilitadores para a aplicação da gestão do conhecimento nas organizações assim como as possíveis barreiras.

O conhecimento não pode ser dissociado das pessoas de uma organização. Desta forma, é preciso criar uma cultura que favoreça o compartilhamento de informações, colaboração e comunicação entre as pessoas. Em estudos realizados por Zheng et al. (2010), a aplicação da gestão do conhecimento é efetiva para a organização quando está alinhada com a estratégia, estrutura e cultura organizacional, sendo esta última o fator mais relevante.

Segundo Wei e Miraglia (2017), a cultura é fator determinante para a implementação da gestão de conhecimento. Os elementos culturais atuam em vários níveis organizacionais podendo apoiar, legitimar, sendo um facilitador, ou por outro lado, desencorajar ações e interações entre indivíduos no compartilhamento de informações e conhecimentos tornando-se uma barreira à aplicação da gestão do conhecimento.

A cultura organizacional afeta positivamente a gestão do conhecimento a partir da existência de um clima colaborativo entre os funcionários baseados em confiança mútua e onde a criação de valor de serviço é o principal objetivo das práticas de gestão de conhecimento. Neste cenário, os indivíduos organizacionais assumem a responsabilidade de sua própria aprendizagem. (HANDZIC, 2011)

Segundo Davenport e Prusak (1999), diversas empresas de consultoria têm utilizado o compartilhamento de conhecimento como forma de critério nas avaliações de desempenho, aliado a práticas de motivação e valorização deste comportamento. A inclusão de critérios voltados ao compartilhamento de conhecimento nas avaliações é um motivador pessoal na medida em que são transformados em estímulo de desenvolvimento pessoal e da carreira profissional.

Para Inkinen (2016), treinamento e um sistema de recompensas robusto são potenciais habilitadores para bons resultados de aceitação às práticas de gestão do conhecimento aplicados em uma organização. Da mesma forma, Paroutis e Saleh (2009) apontam que recompensas, na forma de reconhecimento, são fatores habilitadores para o compartilhamento de conhecimento. Reconhecimento na forma de apreciação não monetária pode ser utilizada de forma estratégica com o objetivo de trazer benefícios de longo prazo para o conhecimento organizacional. (JAIN; MORENO, 2015)

Por outro lado, Bock e Kim (2002) acreditam que sistemas de recompensas, por 
serem motivadores extrínsecos, não são capazes de criar um compromisso duradouro a partir de ações e atitudes para o compartilhamento de conhecimento. Pelo contrário, esses incentivos seriam apenas facilitadores, mas são ineficazes para criar uma mudança de comportamento de forma perene na organização.

Outro fator facilitador considerado relevante foi o papel das lideranças engajadas nas diretrizes de gestão de conhecimento das organizações como meio de apoiar, incentivar e criar meios para a aplicação das práticas de criação, compartilhamento e disseminação de conhecimento e informação de forma eficaz e efetiva. (CHAWLA; JOSHI, 2010)

Para Corfield e Paton (2016), a incentivar as lideranças locais, que possuem atitudes encorajadores e que estejam interessadas e comprometidas com a gestão do conhecimento é um meio eficaz de penetração das práticas entre os diversos setores da organização.

Segundo Stewart (1998), as lideranças comprometidas com a gestão do conhecimento organizacional são mais relevantes que qualquer sistema de recompensas para a motivação do compartilhamento do conhecimento pois são referências em seus setores e influenciam por meio do exemplo.

A evolução tecnológica permitiu o armazenamento e a comunicação do conhecimento em formatos eletrônicos sendo traduzido nos sistemas de informação e conhecimento utilizado pelas organizações. (GARCÍA-HOLGADO; GARCÍA-PEÑALVO, 2016)

A infraestrutura tecnológica como apoio a gestão do conhecimento é fundamental, principalmente em organizações de grande porte e com unidades descentralizadas geograficamente. Segundo Handzic (2011), a estrutura tecnológica na organização pode facilitar a aplicação dos processos de conhecimento, fornecendo uma plataforma de compartilhamento de informações e conhecimento a apoio a colaboração entre os indivíduos organizacionais.

Para Chong et al. (2011), os componentes tecnológicos, como tecnologias para compartilhamento do conhecimento e de comunicação são considerados os habilitadores mais relevantes para uma gestão do conhecimento eficaz.

Segundo Sandhu et al. (2011), a maior barreira individual para o compartilhamento de conhecimento nas organizações é a falta de tempo. Da mesma forma, Massingham (2015) atribui à falta de tempo dos indivíduos organizacionais se dedicarem ao desenvolvimento, teste e aplicação das práticas de gestão de conhecimento por dedicarem todo o seu tempo com as suas atividades rotineiras de trabalho.

Para Davenport e Prusak (1999), o tempo é o recurso corporativo mais escasso, do qual é impossível obter uma substituição, embora seja essencial para a geração do conhecimento. Aliado a isso, a falta de reconhecimento por partes dos gerentes da importância para as atividades de gestão do conhecimento como necessárias para o sucesso corporativo.

Para Amayah (2013), o baixo grau de empatia entre os indivíduos é considerado uma barreira relevante ao compartilhamento de conhecimento na organização.

Para Davenport e Prusak (1999), a confiança é um dos principais fatores que interferem na eficiência dos processos ou iniciativas voltados ao compartilhamento de conhecimentos. Sem o fator confiança, qualquer iniciativa nesta temática estará fadada ao fracasso. A confiança está diretamente conectada ao contato pessoal. Iniciativas como reuniões face a face e ambientes de integração entre os funcionários são exemplos de facilitadores para o desenvolvimento de confiança entre as partes.

Nonaka e Takeuchi (2008, p. 99) definiram Ba como um "contexto compartilhado em movimento, no qual o conhecimento é partilhado, criado e utilizado". O Ba pode ser entendido como um contexto capacitante, podendo ser físico, virtual ou mental. 
São os espaços compartilhados que encorajam e estimulam a interação dos indivíduos e promove a convivência. A ausência desses espaços compartilhados, seja físico ou virtual, é uma barreira a interação dos indivíduos dentro da organização dificultando o fluxo do conhecimento. Para Sandhu at al. (2011) esta barreira se apresenta como a falta de interação entre aqueles que necessitam de determinado conhecimento e aqueles que detém o conhecimento na organização.

\subsection{FERRAMENTAS E PRÁTICAS DE GESTÃO DO CONHECIMENTO}

A seguir serão apresentadas algumas ferramentas e práticas para a aplicação da gestão do conhecimento nas organizações. Entretanto, a utilização de ferramentas não é a base da gestão do conhecimento e sim a plena utilização da gestão do conhecimento nos processos organizacionais e estes devidamente alinhados com a estratégia da organização.

Segundo Batista (2012, p. 81), práticas de gestão do conhecimento são "práticas de gestão voltadas para produção, retenção, disseminação, compartilhamento e aplicação do conhecimento dentro das organizações, bem como na relação dessas com o mundo exterior”.

Para Grimaldi e Rippa (2011), as ferramentas de gestão do conhecimento capturar, organizar, compartilhar e alavancar elementos da gestão do conhecimento, mas necessitam de suporte e treinamento para que sua utilização seja eficaz para a organização.

Os portais de conhecimento corporativos são amplamente utilizados para apoiar a gestão do conhecimento nas organizações na medida que auxiliam a comunicação, compartilhamento de informações e conhecimento e promoção de colaboração entre indivíduos. Esses portais, são exemplos de ferramentas de Tecnologia da Informação e Comunicação (TIC). As TIC são recursos tecnológicos que promovem comunicação das mais diversas formas e apoiam os processos organizacionais. Segundo Silva e Sousa (2015), os portais corporativos possibilitam a convergência de diversas TIC, tais como ferramenta de colaboração e apoio a decisão, em uma interface única e facilitando a explicitação do conhecimento tácito; o armazenamento, a disseminação e a recuperação do conhecimento. Com o aumento expressivo da quantidade de dados e informações utilizadas pelas organizações, os portais de conhecimento corporativo habilitam uma gestão de conhecimento mais eficiente e eficaz. Nos portais de conhecimento corporativo, várias práticas de gestão do conhecimento podem ser integradas em um único lugar, além de possibilitar a integração entre os diversos usuários da organização.

Os repositórios de conhecimento alocam as principais saídas dos processos de conhecimentos. Podem abrigar os conhecimentos explícitos presentes documentos organizacionais como manuais e procedimentos e lições aprendidas (HANDZIC, 2011). Para Davenport e Prusak (1999) o objetivo dos repositórios de conhecimento é alocar informações e conhecimento presentes em diversos documentos em um repositório onde possa ser facilmente armazenado e recuperado.

As Comunidades de Práticas (CoP) são grupos de pessoas que compartilham um interesse comum e que interagem regularmente como forma de inovar e compartilhar conhecimentos. (MASSINGHAM, 2014; WENGER; SNYDER, 2000). Na criação de conhecimento, os ambientes de grupos, a exemplo das comunidades de práticas, estão em um processo de construção coletiva, onde podem ser identificados os processos de socialização e combinação do conhecimento onde os indivíduos disseminam conhecimentos tácitos e explícitos, dando origem a novos conhecimentos por meio da troca de matérias e experiências. (REZENDE; OLIVEIRA; PEREIRA, 2016). As comunidades de práticas podem acontecer de forma presencial ou mesmo de forma virtual, a partir de portais de conhecimentos ou outras ferramentas de tecnologia da informação e comunicação.

Storytelling ou narrativas são construções colaborativas de histórias. Possui estrutura de narrativa ajudando equipes compartilhar elementos do conhecimento tácito. 
(PERRET; BORGES; SANTORO, 2004). Segundo Sanne (2008), storytelling é uma prática bem utilizada por técnicos para a disseminação de aprendizado de incidentes passados.

Uma das práticas de maior potencial para compartilhamento de conhecimento tácito de alta complexidade é o mentoring. Segundo Khajouei e Khajouei (2017), o mentoring é uma prática de trabalho estabelecida entre um mentor (funcionário sênior da organização) e o mentorado com esquema planejado para o compartilhamento de conhecimento a fim de desenvolver a carreira do mentorado. Para Massingham (2014), mentoring é uma ferramenta eficaz para o compartilhamento de experiências entre funcionários que estão na iminência de se desligar da organização e o funcionários que permanecerão no exercício daquela função.

A prática de coaching, segundo Batista (2012), é semelhante ao mentoring, mas o coach não participa da execução das atividades. Tem função de orientação e apoio. Em muitas organizações, esta prática é utilizada para facilitar ambientação e integração de novos funcionários no ambiente e rotina de trabalho.

Segundo Batista (2012), as lições aprendidas são relatos de experiências de processos ou projetos onde são registrados como os fatos aconteceram e o que era esperado que acontecesse e o que foi aprendido durante o processo ou projeto. As lições aprendidas se referem a acontecimentos abaixo do esperado (fator negativo), assim como a acontecimentos acima do esperado (fator positivo) o que pode evidenciar uma possível melhoria de processos. Para Stewart (1998), as lições aprendidas são checklists do que deu certo e o que deu errado em um projeto em conjunto com diretrizes para alavancar projetos similares que aconteçam no futuro.

A quantidade de conhecimento em uma organização é tão grande que seria inviável codificá-la por completo. Neste sentido, os mapas de conhecimento se tornam os orientadores de como chegar aos especialistas em determinado assunto. Segundo Batista (2012), mapas do conhecimento são levantamentos realizados sobre os ativos da organização. Responde a perguntas tais como: quem sabe sobre este assunto, e onde está localizado. Já as páginas amarelas ou banco de talentos, são repositórios sobre as informações sobre a capacitação técnica e científica dos funcionários.

Muito utilizada pelos setores de recursos humanos, as entrevistas de saída, são tradicionalmente realizadas com funcionários que deixam as organizações de forma voluntária. Levantam informações básicas sobre a motivação para a saída entre outras informações de carreira. Entretanto podem ser uma prática absorvida pela gestão do conhecimento na medida em que também podem focar, além de informações básicas da carreira em conhecimentos e informações relativos a função, sendo assim uma forma de compartilhamento de conhecimentos tácitos (FINDLAY, 2016). Segundo Massingham (2015) as entrevistas de saída para a aplicação na gestão do conhecimento podem ser conduzidas e organizadas em temas chave e apresentadas transcritas aos indivíduos envolvidos (titular e sucessor) na atividades em referência.

Recentemente, várias organizações têm estabelecido em sua estrutura as Universidades Corporativas (UC). Segundo Rademakers (2005), as UC são verdadeiros veículos de transferência integrada de conhecimento e desenvolvimento de inovação dentro das organizações. É uma forma institucionalizada de treinamento e capacitação dentro das organizações.

O mapeamento e modelagem de processos, apesar de não ser uma ferramenta originária da gestão de conhecimento é altamente relevante nos processos de conversão do conhecimento. Segundo Cerqueira (2010), o mapeamento de processos apresenta o relacionamento entre entradas, tarefas e saídas. A modelagem do processo geralmente é apresentada em forma de diagrama de caixas facilitando a visualização do fluxo das atividades. O mapeamento é um meio de identificar, registrar, analisar e propor melhorias aos processos. 
Diante disso, o mapeamento e modelagem de processos é uma ferramenta que permite a externalização, compartilhamento e internalização de conhecimentos. Possibilita a transformação do conhecimento informal para o formal. O processo mapeado tem potencial para melhorar a qualidade do conhecimento internalizado, além de aumentar a capacidade de reutilização do conhecimento. (KALPIČ; BERNUS, 2006)

A utilização do sistema de gestão por competências na gestão do conhecimento ou complementar a ela, no contexto de desenvolvimento de competências, tem se tornado cada vez mais comum entre as organizações. Segundo Batista (2012), na gestão por competência são analisadas as competências (conhecimentos, habilidades e atitudes) organizacionais necessárias em comparação com as competências atuais dos indivíduos da organização. As lacunas de competência observadas devem ser desenvolvidas ou captadas.

Para Carbone et al. (2009), A gestão por competências é um modelo de gestão que visa o planejamento, avaliação e desenvolvimentos nos níveis individual e organizacional das competências necessárias para a realização de atividades e consecução de objetivos.

\section{RESULTADOS}

A partir da revisão da literatura, foram correlacionadas afirmativas que irão compor o instrumento de diagnóstico no formato de um questionário com o embasamento da literatura correspondente. O questionário possui 15 questões. Deste total, 14 questões possuem escala likert com 5 escores (questões de $n^{0} 1$ a 14): discordo totalmente; discordo parcialmente; não discordo nem concordo; concordo parcialmente; concordo totalmente. E uma questão no formato de lista de opções (questão de $\mathrm{n}^{0} 15$ ).

Para o AMRJ, a proposta é que o instrumento seja aplicado aos encarregados dos processos críticos da OM. Estes processos já estão identificados na Organização.

O questionário busca responder às questões referente à percepção dos encarregados dos processos críticos quanto da aplicação da gestão do conhecimento na OM; a percepção dos encarregados dos processos críticos quanto as principais dificuldades para a aplicação da gestão do conhecimento na OM; e a percepção dos encarregados dos processos críticos quanto a aplicabilidade de ferramentas e práticas de gestão do conhecimento.

\begin{tabular}{|c|c|c|}
\hline QUESTIONÁRIO (Escala Likert) & TEMÁTCA & $\begin{array}{c}\text { FUNDAMENTAÇÃO } \\
\text { TEÓRICA }\end{array}$ \\
\hline $\begin{array}{l}\text { Q1) A gestão do conhecimento através de } \\
\text { atividades para a criação, desenvolvimento, } \\
\text { registro, armazenamento, compartilhamento de } \\
\text { conhecimentos é importante para a organização. }\end{array}$ & $\begin{array}{l}\text { Importância da } \\
\text { GC para a } \\
\text { organização }\end{array}$ & $\begin{array}{lcc}\text { Davenport } & \text { e } & \text { Prusak } \\
\text { (1999); } & \text { Nonaka } \\
\text { Takeuchi } & (2008) ; & \text { Stewart } \\
\text { (1998); De } & \text { Angelis } \\
\text { (2013); Chawla e Joshi } \\
\text { (2010); Handzic } & \text { (2011); } \\
\text { Kim e Lee (2010) } & \\
\end{array}$ \\
\hline $\begin{array}{l}\text { Q2) A natureza burocrática de Instituições } \\
\text { públicas, aliada a estrutura rígida hierarquizada } \\
\text { das Organizações Militares dificultam a aplicação } \\
\text { da gestão do conhecimento na Organização. }\end{array}$ & a & $\begin{array}{l}\text { Amayah (2013); Bem et } \\
\text { al. (2013); Nonaka e } \\
\text { Takeuchi (2008) }\end{array}$ \\
\hline $\begin{array}{l}\text { Q3) A efetiva aplicação da gestão do } \\
\text { conhecimento na OM pode minimizar ou absorver } \\
\text { efeitos da perda de capital humano, nem sempre } \\
\text { com a devida reposição e a rotatividade de } \\
\text { pessoal. }\end{array}$ & $\begin{array}{l}\text { Perda de } \\
\text { capital humano }\end{array}$ & $\begin{array}{l}\text { Pee e Kankanhalli (2016); } \\
\text { Salleh (2013) }\end{array}$ \\
\hline
\end{tabular}




\begin{tabular}{|c|c|c|}
\hline $\begin{array}{l}\text { Q4) A resistência a mudanças aliada a outras } \\
\text { características da cultura da OM são fatores } \\
\text { complicadores para a aplicação da gestão do } \\
\text { conhecimento na organização. }\end{array}$ & $\begin{array}{l}\text { Resistência a } \\
\text { mudanças e } \\
\text { cultura } \\
\text { organizacionais } \\
\text { como barreira a } \\
\text { GC }\end{array}$ & $\begin{array}{l}\text { Campos e Baptista (2011); } \\
\text { Nonaka e Takeuschi } \\
\text { (2008); Wei e Miraglia } \\
\text { (2017); Zheng et al. (2010) }\end{array}$ \\
\hline $\begin{array}{l}\text { Q5) A utilização de recompensas, na forma de re- } \\
\text { conhecimento, pode facilitar o engajamento dos } \\
\text { funcionários nas diversas práticas de gestão do co- } \\
\text { nhecimento da OM. }\end{array}$ & Recompensas & $\begin{array}{l}\text { Inkinen (2016); Paroutis e } \\
\text { Saleh (2009); Jain e } \\
\text { Moreno (2015) }\end{array}$ \\
\hline $\begin{array}{l}\text { Q6) As lideranças da OM nos diversos níveis es- } \\
\text { tão comprometidas e interessadas na aplicação da } \\
\text { gestão do conhecimento na Organização. }\end{array}$ & $\begin{array}{l}\text { Papel das } \\
\text { lideranças }\end{array}$ & $\begin{array}{l}\text { Chawla e Joshi (2010); } \\
\text { Corfield e Paton (2016); } \\
\text { Stewart (1998) }\end{array}$ \\
\hline $\begin{array}{l}\text { Q7) Os sistemas de comunicação e informação na } \\
\text { OM são suficientes para apoiar tecnologicamente } \\
\text { a aplicação da gestão do conhecimento. }\end{array}$ & $\begin{array}{l}\text { Tecnologias da } \\
\text { informação e } \\
\text { comunicação e } \\
\text { GC }\end{array}$ & $\begin{array}{l}\text { García-Holgado e García- } \\
\text { Peñalvo (2016); Handzic } \\
\text { (2011); Chong et al. } \\
\text { (2011) }\end{array}$ \\
\hline $\begin{array}{l}\text { Q8) A falta de tempo dos funcionários para a } \\
\text { dedicação às atividades de gestão do } \\
\text { conhecimento é uma barreira relevante observada. }\end{array}$ & $\begin{array}{l}\text { Falta de tempo } \\
\text { como barreira a } \\
\text { GC }\end{array}$ & $\begin{array}{l}\text { Sandhu et al. (2011); } \\
\text { Massingham } \quad(2015) ; \\
\text { Davenport e Prusak (1999) }\end{array}$ \\
\hline $\begin{array}{l}\text { Q9) Existem práticas e/ou ferramentas } \\
\text { estruturadas suficientes para criar e desenvolver } \\
\text { conhecimentos relativos ao meu processo. }\end{array}$ & \multirow{6}{*}{$\begin{array}{l}\text { Ferramentas e } \\
\text { práticas de GC }\end{array}$} & \multirow{6}{*}{$\begin{array}{ll}\text { Grimaldi e Rippa } & \text { (2011); } \\
\text { Batista } & (2012) ; \\
\text { Massingham } & (2015) ; \\
\text { Handzic } & (2011) ; \\
\text { Davenport e } & \text { Prusak } \\
\text { (1999); Silva e Sousa } \\
\text { (2015); Cerqueira } & (2010) ; \\
\text { Kalpic e Bernus } & (2006) ; \\
\text { Carbone et al. (2009) }\end{array}$} \\
\hline $\begin{array}{l}\text { Q10) Existem práticas e/ou ferramentas } \\
\text { estruturadas suficientes para reter conhecimentos } \\
\text { relativos ao meu processo. }\end{array}$ & & \\
\hline $\begin{array}{l}\text { Q11) Existem práticas e/ou ferramentas } \\
\text { estruturadas suficientes para compartilhar } \\
\text { conhecimentos relativos ao meu processo. }\end{array}$ & & \\
\hline $\begin{array}{l}\text { Q12) O meu processo está mapeado e com } \\
\text { procedimentos estabelecidos. }\end{array}$ & & \\
\hline $\begin{array}{l}\text { Q13) Não há dificuldades na busca por } \\
\text { informação e conhecimentos relativos a } \\
\text { procedimentos, manuais, normas existentes na } \\
\text { Organização assim como informações de projetos } \\
\text { já realizados. }\end{array}$ & & \\
\hline $\begin{array}{l}\text { Q14) Existem práticas e/ou ferramentas } \\
\text { estruturadas para a identificação de saídas } \\
\text { programadas de funcionários do meu processo / } \\
\text { setor (cursos de carreira/ aposentadorias/ término } \\
\text { do tempo de contrato de militares temporários) }\end{array}$ & & \\
\hline
\end{tabular}


Q15) Dentre as ferramentas e práticas listadas, assinale com um " $\mathrm{x}$ " aquelas que você considera mais adequadas a sua Organização.

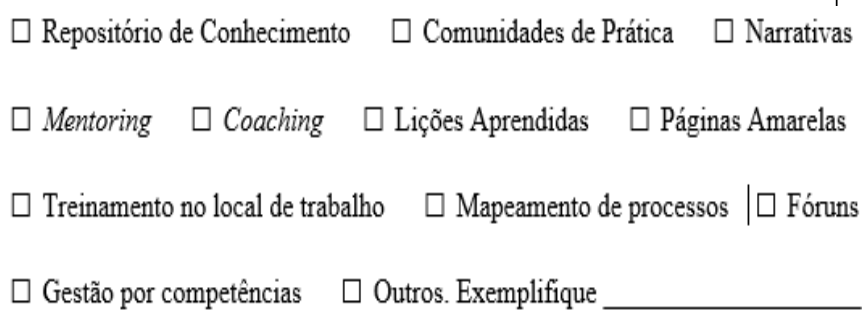

Quadro 2: Instrumento de diagnóstico da GC baseado na literatura Fonte: As autoras

\section{CONCLUSÃO}

Este artigo teve como propósito a elaboração de um instrumento de diagnóstico da Gestão do Conhecimento embasado na literatura para a aplicação no Arsenal de Marinha do Rio de Janeiro. Entretanto pode ser utilizado em outras organizações militares.

A fundamentação do instrumento de pesquisa, visa garantir a validade do instrumento apoiando o desenho das questões em situações ou contextos já relatados na literatura e não baseadas em um reflexo de crenças do pesquisador.

O diagnóstico a ser realizado a partir dos resultados obtidos do questionário proposto neste artigo visa a identificação de lacunas na gestão de conhecimento na organização podendo ser objeto de melhorias ou ponto de partida para a implementação de práticas ou ferramentas de Gestão do Conhecimento.

Como proposta de estudos futuros está a elaboração de um plano de ação visando mitigar ou eliminar os principais problemas que possam ser identificados a partir da aplicação deste instrumento de diagnóstico da gestão do conhecimento organizacional.

\section{REFERÊNCIAS BIBLIOGRÁFICAS}

[1] AMAYAH, A. T. Determinants of knowledge sharing in a public sector organization. Journal of Knowledge Management, v. 17, n. 3, p. 454-471, 2013.

[2] AZEREDO, M. F.; COSTA, S. R. R. da. Gestão do Conhecimento nas Organizações Através da Socialização da Informação. In: CONGRESSO NACIONAL DE EXCELÊNCIA EM GESTÃO \& V INOVARSE, 2018.

[3] BATISTA, F. F. Modelo de gestão do conhecimento para a administração pública brasileira: como implementar a gestão do conhecimento para produzir resultados em benefício do cidadão. Brasília: Ipea, 2012.

[4] BEM, R. M.; PRADO, M. L.; DELFINO, N. Desafios à implantação da gestão do conhecimento: A questão cultural nas organizações públicas federais brasileiras. Revista Digital de Biblioteconomia e Ciência da Informação, v. 11, n. 2, p. 123135, 2013.

[5] BOCK, G. W.; KIM, Y.-G. Breaking the Myths of Rewards: An Exploratory Study of Attitudes about Knowledge Sharing. Information Resources Management Journal (IRMJ), v. 15, n. 2, p. 14-21, 2002. 
[6] CAMPOS, M. M.; BAPTISTA, S. G. Gestão do conhecimento organizacional na administração pública federal. Revista Ibero-Americana de Ciência da Informação, v. 1, n. 1, 9 fev. 2011. Disponível em: <http://periodicos.unb.br/index.php/RICI/article/ view/2015>. Acesso em: 17 out. 2018.

[7] CARBONE, P. P. et al. Gestão por competências e gestão do conhecimento. 3. ed. Rio de Janeiro: Editora FGV, 2009.

[8] CERQUEIRA, J. P. Sistemas de Gestão Integrados: ISO 9001, NBR 16001, OHSAS 18001, SA 8000: Conceitos e aplicações. 2. ed. Rio de Janeiro: Qualitmark, 2010.

[9] CHAWLA, D.; JOSHI, H. Knowledge management initiatives in Indian public and private sector organizations. Journal of Knowledge Management, v. 14, n. 6, p. 811827, 2010.

[10] CHONG, S. C. et al. KM implementation in a public sector accounting organization: An empirical investigation. Journal of Knowledge Management, v. 15, n. 3, p. 497512, 2011.

[11] CHOO, C. W. A Organização do conhecimento: como as organizações usam a informação para criar significado, construir conhecimento e tomar decisões. São Paulo: SENAC, 2003.

[12] CORFIELD, A.; PATON, R. Investigating knowledge management: can KM really change organisational culture? Journal of Knowledge Management, v. 20, n. 1, p. 88-103, 2016.

[13] DAVENPORT, T. H.; PRUSAK, L. Conhecimento Empresarial. Rio de Janeiro: Campus, 1999.

[14] DE ANGELIS, C. T. A Knowledge Management and Organizational Intelligence Model for Public Administration. International Journal of Public Administration, v. 36, n. 11, p. 807-819, 2013.

[15] _ . The impact of national culture and knowledge management on governmental intelligence. Journal of Modelling in Management, v. 11, n. 1, p. 240-268, 2016.

[16] FINDLAY, J. 'Cockroaches of Human Resources Practice'?: Exit Interviews and Knowledge Management. Business Information Review, v. 20, n. 3, p. 127-135, 24 jun. 2016.

[17] FINK, A. Conducting Research Literature Reviews: From the Internet to Paper. Sage: 2005.

[18] GARCÍA-HOLGADO, A.; GARCÍA-PEÑALVO, F. J. Architectural pattern to improve the definition and implementation of eLearning ecosystems. Science of Computer Programming, v. 129, p. 20-34, 2016.

[19] GIL, A. C. Como elaborar projetos de pesquisa. São Paulo: Atlas, 2010.

[20] GRAY, D. E. Pesquisa no mundo real. 2. ed. Porto Alegre: Penso, 2012.

[21] GRIMALDI, M.; RIPPA, P. An AHP-based framework for selecting knowledge management tools to sustain innovation process. Knowledge and Process Management, v. 18, n. 1, p. 45-55, 2011. 
[22] HANDZIC, M. Integrated socio-technical knowledge management model: An empirical evaluation. Journal of Knowledge Management, v. 15, n. 2, p. 198-211, 2011.

[23] INKINEN, H. Review of Empirical Research on Knowledge Management Practices and Firm Performance. Journal of Knowledge Management, v. 20, n. 2, p. 230-257, 4 abr. 2016.

[24] JAIN, A. K.; MORENO, A. Organizational learning, knowledge management practices and firm's performance: An empirical study of a heavy engineering firm in India. Learning Organization, v. 22, n. 1, p. 14-39, 2015.

[25] KALPIČ, B.; BERNUS, P. Business process modeling through the knowledge management perspective. Journal of Knowledge Management, v. 10, n. 3, p. 40-56, 1 maio 2006.

[26] KHAJOUEI, H.; KHAJOUEI, R. Identifying and prioritizing the tools/techniques of knowledge management based on the Asian Productivity Organization Model (APO) to use in hospitals. International Journal of Medical Informatics, v. 108, p. 146151, 2017.

[27] KIM, S.; LEE, H. Factors affecting employee knowledge acquisition and application capabilities. Asia-Pacific Journal of Business Administration, v. 2, n. 2, p. 133-152, 2010.

[28] MASSINGHAM, P. An evaluation of knowledge management tools: Part 1 managing knowledge resources. Journal of Knowledge Management, v. 18, n. 6, p. 1075-1100, 2014.

[29] _. Knowledge Sharing: What Works and What Doesn't Work: A Critical Systems Thinking Perspective. Systemic Practice and Action Research, v. 28, n. 3, p. 197-228, 2015.

[30] NONAKA, I.; TAKEUCHI, H. Gestão do Conhecimento. Porto Alegre: Bookman editora, 2008.

[31] PAROUTIS, S.; SALEH, A. A. Determinants of knowledge sharing using Web 2.0 technologies. Journal of Knowledge Management, v. 13, n. 4, p. 52-63, 2009.

[32] PEE, L. G.; KANKANHALLI, A. Interactions among factors influencing knowledge management in public-sector organizations: A resource-based view. Government Information Quarterly, v. 33, n. 1, p. 188-199, 2016.

[33] PERRET, R.; BORGES, M. R. S.; SANTORO, F. M. Applying group storytelling in knowledge management. Lecture Notes in Computer Science, v. 3198, p. 34-41, 2004.

[34] POLANYI, M. The Tacit Dimension. New York: Doubleday, 1966.

[35] RADEMAKERS, M. Corporate universities: Driving force of knowledge innovation. Journal of Workplace Learning, v. 17, p. 130-136, 1 jan. 2005.

[36] REZENDE, J. F.; OLIVEIRA, D. A.; PEREIRA, R. D. Plataformas para gestão do conhecimento: Estudo de caso sobre a ativação do valor de excedentes cognitivos por meio do desenvolvimento de um contexto capacitante virtual. Perspectivas em Gestão \& Conhecimento, v. 6, n. 1, p. 72-88, 2016. 
[37] RIBEIRO, C. P. D. P. et al. Dissemination of information within the public sector. Transinformacao, v. 23, n. 2, p. 159-171, 2011.

[38] ROCHA, E. S. B. et al. Gestão do conhecimento na saúde: revisão sistemática de literatura. Rev. Latino-Am. Enfermagem, Ribeirão Preto, v. 20, n. 2, p. 392400, 2012.

[39] SALLEH, K. Knowledge sharing and knowledge management modelling in public sector accounting organisation: Case evidence from Malaysia. International Journal of Knowledge-Based Development, v. 4, n. 4, p. 363-381, 2013.

[40] SANDHU, M. S.; JAIN, K. K.; AHMAD, I. U. K. b. Knowledge sharing among public sector employees: Evidence from Malaysia. International Journal of Public Sector Management, v. 24, n. 3, p. 206-226, 2011.

[41] SANNE, J. M. Incident Reporting or Storytelling? Competing Schemes in a SafetyCritical and Hazardous Work Setting. Safety Science, v. 46, n. 8, p. 1205-1222, out. 2008.

[42] SIGUENZA-GUZMAN, L. et al. Literature Review of Data Mining Applications in Academic Libraries. The Journal of Academic Librarianship, v. 41, n. 4, p. 499510, 1 jul. 2015.

[43] SILVA, N. B. X.; SOUSA, M. R. F. de. A dimensão tecnológica da gestão do conhecimento e a contribuição da arquitetura da informação: Uma análise da plataforma podio. Perspectivas em Gestão \& Conhecimento, v. 5, n. 2, p. 186-200, 2015.

[44] STEWART, T. A. Capital Intelectual: A Nova Vantagem Competitiva das Empresas. Rio de Janeiro: Campus, 1998.

[45] WEI, Y.; MIRAGLIA, S. Organizational Culture and Knowledge Transfer in ProjectBased Organizations: Theoretical Insights from a Chinese Construction Firm. International Journal of Project Management, v. 35, n. 4, p. 571-585, maio 2017.

[46] WENGER, E. C.; SNYDER, W. M. Communities of Practice: The Organizational Frontier. Harvard Business Review, v. 71, n. 1, p. 139-145, 1 jan. 2000.

[47] ZHENG, W.; YANG, B.; MCLEAN, G. N. Linking Organizational Culture, Structure, Strategy, and Organizational Effectiveness: Mediating Role of Knowledge Management. Journal of Business Research, v. 63, n. 7, p. 763-771, jul. 2010. 